Artículo

\title{
Rendimiento de semilla y calidad de fruto de chile habanero con fertilización química y orgánica
}

\author{
Mariano Mendoza-Elos ${ }^{1}$ \\ Luis Felipe Zamudio Alvarez ${ }^{1}$ \\ Francisco Cervantes Ortiz ${ }^{1}$ \\ Francisco Chable Moreno ${ }^{1}$ \\ Jesús Frías Pizano ${ }^{1}$ \\ Alfredo Josué Gámez Vázquez ${ }^{2 \S}$ \\ ${ }^{1}$ Tecnologico Nacional de México-Instituto Tecnológico de Roque. Carretera Celaya-Juventino Rosas km \\ 8. Roque, Celaya, Guanajuato, México. CP. 38110. (mmendoza66@hotmail.com; felipe- \\ zamudio@hotmail.com; $\quad$ garcia-2956@yahoo.com.mx; $\quad$ frcervantes@itroque.edu.mx; \\ fchable4oct@hotmail.com; jesus-friasp@yahoo.com.mx). ${ }^{2}$ Campo Experimental Bajío-INIFAP. Carretera \\ Celaya-San Miguel de Allende km 6.5, Celaya, Guanajuato, México. CP. 38110.

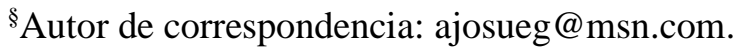

\section{Resumen}

El objetivo de la presente investigación consistió en evaluar poblaciones de chile habanero, con fertilización química y orgánica, para rendimiento de semilla y fruto. En 2017 en el Tecnológico Nacional de México campus Roque se establecieron tres genotipos de chile habanero con cinco diferentes tipos de fertilización. Un grupo de variables agronómicas se evaluaron con las normas del SNICS. Las variables se sometieron a un análisis de varianza y componentes principales. La mejor colecta fue el genotipo Rojo Campeche 12 con un peso promedio de fruto de $4.8 \mathrm{~g}, 27.5$ semillas por fruto y una germinación a los 16 días de 39.2\%. Con relación a la fertilización se determinó que el mejor peso de fruto se alcanzó con el 50+50\% de fertilización química y orgánica. Para semillas por fruto y germinación fue mejor la fertilización química, aunque estadísticamente similar al valor de la fertilización combinada $(50+50)$. Lo anterior, permite explorar genotipos, dosis y oportunidades de aplicación de abonos orgánicos que mejoren la producción y calidad de semilla, con manejo sustentable y amigable con el ambiente.

Palabras clave: Capsicum chinense Jacq., germinación, producción orgánica.

Recibido: agosto de 2020

Aceptado: noviembre de 2020 


\section{Introducción}

La producción de chile habanero ha sido limitada por factores como la incidencia de plagas y enfermedades, programación deficiente del riego y la nutrición, lo cual es grave bajo condiciones de invernadero, donde con un adecuado manejo, se reduce la probabilidad de infestaciones por enfermedades y plagas; además de no limitar la producción del cultivo y calidad del fruto, así se ha observado que el chile habanero responde apropiadamente a la aplicación de nitrógeno, incrementando rendimiento y su calidad nutricional (Huez, 2013).

Entre la gran diversidad del género Capsicum, el chile habanero ( $C$. chinense Jacq.) se ha convertido en un símbolo y ejemplo en pungencia, debido a su alto contenido de capsaicina en el fruto. La importancia de los capsaicinoides se debe a que además de proporcionar el sabor picante, son utilizados por la industria farmacéutica, de armas, tabacalera, cosmética y de pinturas, entre otras, dando lugar a diversos productos (Borges et al., 2014). Se estima que, de la producción total, $75 \%$ se destina para el consumo en estado fresco, $22 \%$ lo utiliza la industria en la elaboración de salsas y el 3\% se destina a la obtención de semilla (Tucuch et al., 2012).

Respecto a la comercialización de chile habanero en fresco, SIAP (2015) reportó que el precio más alto al productor se localizó en Nayarit con \$29.41, el precio más bajo se situó en Campeche con \$9.61, mientras que el precio al consumidor más alto se ubicó en Durango, con \$99.90 y el precio más bajo se detectó en Veracruz, con $\$ 29.75$. La industria para salsa da preferencia a ciertas variedades y color, siendo estos rojos y amarillos (Huez, 2013).

En el sureste mexicano el chile habanero ha incrementado su superficie sembrada, en 2014 se establecieron 951.52 ha, con una producción de 9977 t (SIAP, 2015). Actualmente el chile habanero se cultiva en diversas regiones de México, principalmente en los estados de Yucatán, Tabasco, Campeche y Quintana Roo, con rendimientos que oscilan de 10 a $30 \mathrm{t} \mathrm{ha}^{-1}$, dependiendo del nivel de tecnificación empleado en el cultivo (Latournerie et al., 2015). En 2010 se publicó la denominación de origen como 'Chile habanero de la Península de Yucatán' (DOF, 2010), que dio lugar a la resolución del 'Proyecto de Norma Oficial Mexicana PROY-NOM189-SCFI-2012 Chile habanero de la Península de Yucatán (Capsicum chinense Jacq.)- especificaciones y métodos de prueba' (DOF, 2012).

Las especies hortícolas enfrenta limitantes que minimizan su potencial de rendimiento y utilidad. La nutrición de las plantas es preponderante porque el mal uso de los fertilizantes químicos puede tener un costo económico alto, además de causar efectos adversos al suelo y agua. Una alternativa ecológicamente para aumentar el rendimiento de cultivos es la inoculación de microorganismos promotores del crecimiento, denominados bioestimulantes o biofertilizantes (Reyes et al., 2014).

Los abonos orgánicos son de gran importancia en la fertilización del suelo, esto dependiendo de la naturaleza del abono, características del suelo, tipo de cultivo, periodicidad de la aplicación y cantidad aplicada del abono. Los abonos orgánicos proporcionan nutrientes, corrigen deficiencias porque contienen nutrientes de lenta liberación y mejoran las condiciones físico-químicas y biológicas del suelo, así ayudan al desarrollo y crecimiento de las plantas (López et al., 2012). Además, la aplicación de agroquímicos en la agricultura moderna ha provocado la degradación de recursos naturales y la erosión tecnológica de los sistemas tradicionales de producción, poniendo en riesgo la productividad sustentable de los agroecosistemas (Nieves et al., 2013). 
En la producción comercial de semillas la calidad está determinada por un conjunto de atributos, donde la calidad genética, física, sanitaria y fisiológica juegan un papel importante. La calidad fisiológica implica la integridad de las estructuras y procesos fisiológicos, siendo los principales indicadores: la viabilidad, germinación y vigor, que dependen del genotipo. Entre los factores que pueden tener efecto en la calidad de la semilla están el grado de madurez y tiempo de maduración de la semilla después de la cosecha (Tamayo et al., 2014).

Uno de los retos en la producción del chile habanero es contar con plántulas sanas, vigorosas y de excelente calidad al momento del trasplante. Las fertilizaciones minerales al suelo podrían potencializarse con el uso de compostas, ya que la aplicación frecuente de éstas mejora la estructura del suelo. Para que el sistema de producción de chile habanero sea sustentable, se deben reducir las aplicaciones de fertilizantes químicos que encarecen la cadena productiva de este cultivo.

Por tal motivo, es conveniente realizar aplicaciones de compostas que equilibren la nutrición del cultivo y sea factible a los productores su elaboración. Una estrategia para mejorar la nutrición orgánica es la rotación de cultivos con leguminosas. Aunque algunos abonos orgánicos presentan altas concentraciones de macronutrientes, el análisis de contenidos totales es un referente para definir su valor real como abono orgánico (Ertani, 2015). Por lo anterior, el objetivo de esta investigación fue evaluar el efecto de fertilizantes químicos y orgánicos sobre el rendimiento de semilla, de chile habanero y su calidad de fruto.

\section{Materiales y métodos}

El estudio se realizó en el campo experimental del Tecnológico Nacional de México Campus Roque, Celaya, Guanajuato, con coordenadas geográficas de $20^{\circ} 34^{\prime} 54.24^{\prime \prime}$ latitud norte y $100^{\circ}$ 49' 35.34" longitud oeste, a $1767 \mathrm{~m}$ de altitud. El clima predominante es semicálido, subhúmedo BS y C (Wo) con temperatura media que oscila de los 14 a los $22{ }^{\circ} \mathrm{C}$ y la precipitación pluvial varía de los 600 hasta los $1000 \mathrm{~mm}$ anuales. Los suelos de la región son de textura arcillosa, planos de reacción ligeramente alcalina, sumamente fértiles y aptos para una gran variedad de cultivos.

Para el establecimiento de las semillas de chile habanero se realizó la siembra en charolas germinadoras, esterilizadas con hipoclorito de sodio al $20 \%$ durante $12 \mathrm{~h}$. El sustrato fue una mezcla del número 3 de la marca Sunshine ${ }^{\circledR}$ y vermiculita. Se utilizaron 200 semillas por genotipo de tres colectas, uno de fruto rojo y los otros dos, color naranja.

Previamente, se elaboró la mezcla de sustrato donde se colocaron las plántulas, la proporción fue 1:1:1 de tezontle-tierra-lombricomposta. Se mezclaron y se colocaron en bolsas para invernadero de 25 x $25 \mathrm{~cm}$ y en proporción de $4 \mathrm{k}$ de mezcla de sustrato por bolsa. Se dio un tratamiento pre trasplante con un promotor de desarrollo radical elaborado a base de $\mathrm{N}(7 \%), \mathrm{P}_{2} \mathrm{O}_{5}(47 \%), \mathrm{K}_{2} \mathrm{O}$ (6\%), L-aminoácidos (3\%) ácidos húmicos $(15.5 \%)$, auxinas $(0.03 \%)$ y materia inerte $(21.47 \%)$ $(20 \mathrm{~g})$, un promotor biológico de desarrollo con Pseudomonas fluorescens $\left(1 \mathrm{x} 10^{5} \mathrm{ufc} \mathrm{mL}^{-1}\right)$, Azotobacter spp. $\left(1 \times 10^{5} \mathrm{ufc} \mathrm{ml}^{-1}\right)$, Bacillus spp. $\left(1 \times 10^{5} \mathrm{ufc} \mathrm{ml}^{-1}\right)$ acondicionadores y $16.8 \%$ de diluyentes orgánicos (40 ml), (40 ml) un complejo de nutrientes quelatados a base de $\mathrm{Mg}(1.5 \%)$, carbohidratos (1.5\%) Mn (1.5\%) Fe (3\%) ácidos fúlvicos (1\%) extracto soluble de pescado (5\%) y $86.5 \%$ de acondicionadores y diluyentes $(40 \mathrm{ml})$ y agua $(101)$. Sumergiendo cada cepellón durante cinco segundos. El trasplante se realizó el 24 de marzo de 2017, a los 105 días después de siembra. 
El programa de fertilización química y fertilización orgánica se realizó de acuerdo con lo postulado por Soria (2002); López (2012), respectivamente: fertilización química: 125-100-150, primera fertilización (desarrollo vegetativo): $63 \%$ N - 33\% P - 33\% K. Segunda fertilización (floración y amarre): $25 \% \mathrm{~N}-50 \%$ P- $22 \% \mathrm{~K}$ y la tercera fertilización (fructificación): $12 \% \mathrm{~N}-17 \% \mathrm{P}-45 \% \mathrm{~K}$. Como fertilización orgánica: lombricomposta (2-2-4), $200 \mathrm{~g}$ planta $^{-1}$ cada 20 días, durante todo el ciclo a partir de los 18 días de establecidos.

La fertilización porcentual complementaria de nutrientes químicos y abonos orgánicos utilizados en la producción de chile habanero, fuero cinco combinaciones de fertilización: químico 100\%, químico $75 \%$ + orgánico $25 \%$, químico $50 \%$ + orgánico $50 \%$, químico $25 \%$ + orgánico $75 \%$ y $100 \%$ orgánico.

Los fertilizantes, tanto químico como orgánico, fueron aplicados al sustrato en forma de sólidos. Complementaria a esta fertilización, en todas las unidades experimentales se realizaron tres aplicaciones foliares de microelementos con productos comerciales cuyo contenido es: a) Fe 4\%,

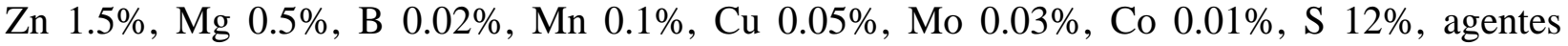
quelatantes $45 \%$, diluyentes y acondicionadores 36.6\% (10 ml); y b) $\mathrm{N} \mathrm{8 \% ,} \mathrm{P}_{2} \mathrm{O}_{5} 4 \%, \mathrm{~K}_{2} \mathrm{O} 3 \%$, $\mathrm{CaO} 0.49 \%$, Zn 0.44\%, Mn 0.4\%, S 0.27\%, Co 0.16\%, MgO 0.09\%, B 0.034\%, Cu 0.01\%, Fe $0.01 \%$, Mo $0.004 \%$, ácidos húmicos $0.005 \%$, fúlvicos $0.2 \%$ y AIA $0.003 \%$ (10 ml), además del complejo de nutrientes quelatados descrito previamente.

Se detectó la presencia de mosca blanca (Bemisia tabaci) y araña de dos puntos (Tetranicus urticae) en las etapas de desarrollo vegetativo y floración. Para su control preventivo, se hicieron aplicaciones semanales de repelentes orgánicos constituidos por extracto de ajo (25\%), chile picante $(25 \%)$ y canela $(10 \%)$, además de un acaricia biológico a base de extracto de canela.

\section{Variables evaluadas}

En la cosecha se evaluó en g el peso de fruto (peso fruto), número de lóculos por fruto (loc fruto ${ }^{-1}$ ) de forma individual, grosor del pericarpio (per fruto ${ }^{-1}$ ) al medir la pared externa del fruto (mm) de forma transversal y el número de semillas por fruto (sem fruto ${ }^{-1}$ ), de acuerdo con SNICS (2019). Previamente los frutos seleccionados se dejaron en reposo durante 15 días, con la finalidad de lograr su madurez fisiológica.

Para el análisis de semillas, se evaluaron con la metodología de ISTA (2004); es decir, con cuatro repeticiones de 25 semillas cada una, para evaluar el porcentaje de semillas que produjeron plántulas normales al octavo (PorGerm8D) y al décimo sexto día (PorGerm16D), (ISTA, 2004).

En invernadero se usó un diseño factorial en bloques completos al azar con tres genotipos, cinco tipos de fertilizaciones y dos repeticiones. La unidad experimental constó de dos bloques de $7 \mathrm{~m}$ de longitud por $6.4 \mathrm{~m}$ de ancho, en una superficie de $89.6 \mathrm{~m}^{2}, 30$ plantas por genotipo, un acomodo entre plantas de $0.6 \mathrm{~m}$ y separación de $0.5 \mathrm{~m}$ entre bloques. Las variables de fruto, semilla y germinación se sometieron a un Anava, la prueba de medias de Tukey $(p=0.05)$ y un análisis de componentes principales, en el SAS ver. 9.3. (SAS, 2003). 


\section{Resultados y discusión}

En el análisis de varianza en la etapa de producción y cosecha de frutos, se detectaron diferencias estadísticas significativas $(p \leq 0.01)$ para peso de fruto, número de lóculos por fruto, grosor del pericarpio por fruto, número de semillas por fruto, y grosor del pericarpio, debidas a los genotipos, tratamientos de fertilización y la interacción de estos. De forma similar, en los análisis de varianza para el porcentaje de germinación a los 8 y 16 días también existieron diferencias significativas $(p \leq 0.01)$ entre tratamientos, genotipos y la interacción de ambos. En todos los Anova la mayor variabilidad detectada fue debida a genotipos, seguida por los tratamientos de fertilización y al final por la interacción.

La fertilización mixta con 50\% de fertilización química y 50\% de orgánica, muestra los valores más altos en peso de fruto ( $4.78 \mathrm{~g})$, número de lóculos por fruto (3), grosor de pericarpio por fruto $(0.21 \mathrm{~cm})$ y número de semillas por fruto $(16.78)$; tratamiento que registró una respuesta superior al de la fertilización totalmente química en el peso de fruto y número de lóculos por fruto. El tratamiento $25 \%$ - $75 \%$ presentó una respuesta similar en todas las variables evaluadas, en comparación con la fertilización totalmente química (0\% - 100\%).

En el caso contrario, el tratamiento 5 (0-100), arroja los índices de menor magnitud: peso de fruto $(1.4 \mathrm{~g})$, número de lóculos por fruto $(2)$, grosor de pericarpio por fruto $(0.11 \mathrm{~cm})$ y número de semillas por fruto (2.56), por lo que los niveles de exploración de cantidad y oportunidad en la fertilización no cubrieron las demandas del cultivo. Siendo estadísticamente diferente al resto de los tratamientos, posicionándose en última escala.

En rendimiento de semilla por fruto se agrupan tres tratamientos en un primer grupo, los valores oscilan de 16.78 a 27.67 semillas por fruto, participan dos tratamientos con fertilización orgánica (Cuadro 1). Estos resultados indican que, la incorporación de abonos orgánicos es muy importantes en la producción de semilla de chile habanero, contrastante, si se desea producir frutos, pues en este caso, los rendimientos en peso de frutos son menores a los tratamientos químicos.

Cuadro 1. Comparación de medias para características de frutos y semillas de chile habanero con fertilización química y orgánica, Roque, 2018.

\begin{tabular}{|c|c|c|c|c|c|c|}
\hline \multirow{2}{*}{ Fertilización } & \multicolumn{2}{|c|}{ Descripción } & \multicolumn{4}{|c|}{ Valor medio } \\
\hline & Químico (\%) & Orgánico (\%) & Peso fruto $(\mathrm{g})$ & Loc fruto $^{-1}$ & Per fruto $^{-1}(\mathrm{~mm})$ & Sem fruto ${ }^{-1}$ \\
\hline 1 & 100 & 0 & $4.61 \mathrm{~b}$ & $2.72 \mathrm{~b}$ & $0.2 \mathrm{ab}$ & $27.67 \mathrm{a}$ \\
\hline 2 & 75 & 25 & $3.08 \mathrm{bc}$ & $1.67 \mathrm{c}$ & $0.12 \mathrm{~b}$ & $9.67 \mathrm{~b}$ \\
\hline 3 & 50 & 50 & $4.78 \mathrm{a}$ & $3 \mathrm{a}$ & $0.21 \mathrm{a}$ & $16.78 \mathrm{a}$ \\
\hline 4 & 25 & 75 & $4.08 \mathrm{~b}$ & $2.67 b c$ & $0.18 \mathrm{~b}$ & $17.94 \mathrm{a}$ \\
\hline 5 & 0 & 100 & $1.4 \mathrm{c}$ & $2 \mathrm{bc}$ & $0.11 \mathrm{~b}$ & $2.56 \mathrm{c}$ \\
\hline
\end{tabular}

Letras iguales significa que no existen diferencias estadísticas. peso fruto= peso por fruto; 1 loc fruto ${ }^{-1}=$ número de lóculos por fruto; per fruto ${ }^{-1}=$ grosor del pericarpio por fruto; sem fruto ${ }^{-1}=$ número de semillas por fruto.

Para genotipos, la comparación de medias muestra diferencias estadísticas significativas entre cada material genético. Mostrando independencia en el agrupamiento individual en todas las variables, a excepción del grosor del pericarpio por fruto, donde la variación es muy estrecha y los materiales 
G19 (Naranja Yucatan-14) y G20 (Naranja Yucatan-15) son similares con valores de 0.15 y 0.14 $\mathrm{cm}$, respectivamente. Con relación a las otras variables, peso de fruto, lóculos por fruto y semillas por fruto, el genotipo 19 supera al 20.

El genotipo Rojo Campeche-12 (G4) muestra los valores más altos en el peso de fruto fue de 4.8 g, número de lóculos por fruto es de 2.97 , grosor del pericarpio por fruto de $0.17 \mathrm{~cm}$ y 27.5 para el número de semillas por fruto, es decir, superó en promedio con $54.4 \%$ en rendimineto por futo al resto de las colectas (Cuadro 2). El efecto del tipo de fertilizacion quimica versus organica y aunado a la constitucion genetica de cada poblacion evaluada de chile habanero, resulta que los dos factores de importancia en esta investigacion cobra diferencias estadisticas para fruto y semilla.

Cuadro 2. Comparación de medias para frutos entre genotipos de chile habanero. Roque, 2018.

\begin{tabular}{cccccc}
\hline \multirow{2}{*}{ Genotipo } & \multirow{2}{*}{ Descripción } & \multicolumn{4}{c}{ Valor medio } \\
\cline { 3 - 6 } & & Peso fruto $(\mathrm{g})$ & Loc fruto $^{-1}$ & Per fruto $^{-1}(\mathrm{~mm})$ & ${\text { Sem } \text { fruto }^{-1}}$ \\
\hline G4 & Rojo-Campeche-12 & $4.8 \mathrm{a}$ & $2.97 \mathrm{a}$ & $0.17 \mathrm{a}$ & $27.5 \mathrm{a}$ \\
G19 & Naranja-Yucatán-14 & $3.27 \mathrm{~b}$ & $2.27 \mathrm{~b}$ & $0.15 \mathrm{~b}$ & $13.17 \mathrm{~b}$ \\
G20 & Naranja-Yucatán-15 & $1.1 \mathrm{c}$ & $1.67 \mathrm{c}$ & $0.14 \mathrm{~b}$ & $4.1 \mathrm{c}$ \\
\hline
\end{tabular}

Letras iguales significa que no existe diferencias estadísticas. Peso fruto $=$ peso por fruto; Loc fruto ${ }^{-1}=$ número de lóculos por fruto; per fruto ${ }^{-1}=$ grosor del pericarpio por fruto; sem fruto ${ }^{-1}=$ número de semillas por fruto.

$\mathrm{Al}$ respecto, López et al. (2017) mencionan que la mejor respuesta en el tamaño y peso del fruto lo obtuvieron con el régimen $14\left(\mathrm{NO}_{3}{ }^{-}\right), 12: 1: 7\left(\mathrm{NO}_{3}: \mathrm{H}_{2} \mathrm{PO}_{4}: \mathrm{SO}_{4}\right)$ y 14:5 $\left(\mathrm{NO}_{3}: \mathrm{K}^{+}\right)$me $\mathrm{L}^{-1}$, logrando en promedio, frutos de $3.48 \mathrm{~cm}$ de longitud, $2.29 \mathrm{~cm}$ de diámetro y un peso de $3.45 \mathrm{~g}_{\text {fruto }}{ }^{-1}$. Datos que difieren por debajo de los presentados en el presente trabajo, donde en el caso específico del genotipo Rojo Campeche-12 (G4) y la fertilización 3 (50\% orgánico y 50\% químico), los pesos medios de los frutos oscilan en los $4.8 \mathrm{~g} \mathrm{fruto}^{-1}$.

De acuerdo con Ramírez et al. (2012), la longitud del fruto de chile habanero, variedad 'Jaguar' es de 3.8 a $5.5 \mathrm{~cm}$, el diámetro de 2.5 a $3 \mathrm{~cm}$ y de 6.5 a $10 \mathrm{~g}_{\text {fruto }}{ }^{-1}$; no obstante, no señalan si ese tamaño de fruto se obtiene bajo condiciones de invernadero. En otro trabajo, Tucuch (2012), menciona que para las variables rendimiento y calidad del fruto (peso, longitud y diámetro) los efectos de la solución nutritiva de manera independiente al sustrato, se observan con tratamiento cuya relación amonio/nitrato fue de 20/80\%.

Lo cual permite afirmar por principio y en forma general que las proporciones de amonio en la solución nutrimental del orden de 10 a 30\% del total de $\mathrm{N}$ y tamaño de gránulo del tezontle del orden de 5 a $20 \mathrm{~mm}$ se asocian a mayores rendimientos de fruto. Estos valores coinciden con lo aquí reportado, donde la mejor calidad de frutos fue obtenida con la fertilización 3 (50-50), cuya aportación de nitrato en forma química disminuye $50 \%$ y la granulometría de tezontle es similar.

También López et al. (2012) observaron diferencias significativas entre los tratamientos para el rendimiento de fruto fresco de chile habanero, las plantas tratadas con lombricomposta y la infusión de estiércol, obtuvieron rendimientos de $949 \mathrm{~g} \mathrm{planta}^{-1}$ y $863 \mathrm{~g} \mathrm{planta}^{-1}$, respectivamente. Las plantas tratadas con composta, bokashi y el testigo obtuvieron rendimientos de 687, 679 y $325 \mathrm{~g} \mathrm{planta}^{-1}$. Los parámetros no pueden ser comparados equitativamente, pues en este proyecto se llevó a cabo un raleo de 20 frutos planta $^{-1}$, obteniendo un peso por fruto que oscila de 1.4 a $4.78 \mathrm{~g}_{\text {fruto }}{ }^{-1}$. 
Por su parte, Quintal et al. (2012) mencionan que el tratamiento con mayor nivel de humedad (T1, $60 \%$ de la humedad aprovechable (HA) fue de mayor rendimiento y peso de frutos con respecto a los otros tratamientos. El menor rendimiento y tamaño de frutos se registraron en T4 y T5, que corresponden a 30 y $20 \%$ de HA. El índice de productividad del agua (IPA) fue mayor $(p \leq 0.05)$ con $60 \%$ de HA, que en T4 y T5, con 30 y $20 \%$ de HA, respuesta que es directamente proporcional con el rendimiento.

La respuesta de los tres genotipos estuvo asociada con su adaptación a las condiciones de temperatura ambiente en las que se evaluaron, que en este caso, no fueron las idóneas para el desarrollo y expresión del genotipo G20 (Naranja Yucatán 15), como lo argumentan Latournerie et al. (2015).

En la comparación de medias respecto al tipo de fertilización se observan dos grupos en el porcentaje de germinación a los 8 días. El primer grupo está conformado por la fertilización 1 (1000), la 3 (50-50) y la 4 (25-75), estos datos oscilan, de 19.3 a 25, los mayores índices de germinación, mostrando con ello, que la aplicación de compostas aporta un alto vigor de la semilla en los diferentes genotipos. En la evaluación del porcentaje de germinación a los 16 días, la disparidad y diferenciación estadística entre tratamientos se torna más notoria al reagruparse en 4 grupos.

Los valores más altos de germinación fueron en la fertilización 1 (100-0) y 3 (50-50) con 40 y $37 \%$, respectivamente. Seguidos por el tratamiento 4 (25-75) cuyos valores intermedios representan un porcentaje de germinación aceptable, no siendo así para los tratamientos 2 (75-25) y 5 (0-100) que muestran un porcentaje bajo y muy bajo en la germinación, respectivamente (Cuadro 3). Por lo anterior, el mejor tratamiento (50-50), da lugar a la producción de semilla sustentable y saludable, ya que además de resultar con la mejor calidad de fruto (peso, número de lóculos y grosor de pericarpio), también presentó el mayor número de semillas y calidad fisiológica de éstas (germinación). En la medida en que se modificó la relación 50:50, se redujo la germinación, por lo que probablemente se alteró la asimilación y translocación de materia seca a la semilla.

Cuadro 3. Comparación de medias para porcentajes de germinación a los 8 y 16 días entre tratamientos en semillas de chile habanero. Roque, 2018.

\begin{tabular}{cccccc}
\hline \multirow{2}{*}{ Tratamiento } & \multicolumn{2}{c}{ Descripción } & & \multicolumn{2}{c}{ Valor Medio } \\
\cline { 2 - 3 } \cline { 5 - 6 } & Químico $(\%)$ & Orgánico $(\%)$ & & PorGerm8D & PorGerm16D \\
\hline 1 & 100 & 0 & & $25 \mathrm{a}$ & $40 \mathrm{a}$ \\
3 & 75 & 25 & & $3 \mathrm{~b}$ & $13 \mathrm{c}$ \\
4 & 50 & 50 & & $20.67 \mathrm{a}$ & $37 \mathrm{a}$ \\
5 & 25 & 75 & & $19.33 \mathrm{a}$ & $30.67 \mathrm{~b}$ \\
\hline
\end{tabular}

Letras iguales significa que no existen diferencias estadísticas. PorGerm8D= porcentaje de germinación a los 8 días; PorGerm16D= porcentaje de germinación a los 16 días.

Para el caso de genotipos, se observa un fuerte efecto genético, siendo estadísticamente diferentes entre ellos, el genotipo Rojo Campeche-12 con los porcentajes de germinación más altos a los 8 y 16 días (26 y 39.2, respectivamente) fue superior al resto. El genotipo con los valores más bajos en germinación fue el Naranja Yucatan-15 (Cuadro 4). Ambos genotipos fueron superados por G4 
con 31.63 y $71.42 \%$ a los 16 días después de la siembra. La variable más importante para medir la calidad fisiológica de una semilla es la germinación en todos los cultivos. En el caso de la semilla de chile habanero su conservación y calidad aún sigue siendo problema, en tanto, se debe estudiar en mayor grado el efecto de su nutrición y su almacenamiento, principalmente. El resultado obtenido alcanzó $39.20 \%$ de germinación a los 16 días después de la siembra.

Cuadro 4. Comparación de medias para porcentajes de germinación a los 8 y 16 días entre genotipos de chile habanero. Roque, 2018.

\begin{tabular}{cccc}
\hline \multirow{2}{*}{ Genotipo } & \multirow{2}{*}{ Descripción } & \multicolumn{2}{c}{ Valor medio } \\
\cline { 3 - 4 } & & PorGerm8D & PorGerm16D \\
\hline G4 & Rojo-Campeche-12 & $26 \mathrm{a}$ & $39.2 \mathrm{a}$ \\
G19 & Naranja-Yucatán-14 & $14.2 \mathrm{~b}$ & $26.8 \mathrm{~b}$ \\
G20 & Naranja-Yucatán-15 & $4.2 \mathrm{c}$ & $11.2 \mathrm{c}$ \\
\hline
\end{tabular}

PorGerm8D= porcentaje de germinación a los 8 días; PorGerm16D= porcentaje de germinación a los 16 días.

Herrera et al. (2018) en un estudio de colectas de chile piquín encontró la mayor tasa de germinación a los 21 después de la siembra, valores que oscilan de 30 a 40\%. Ayala et al. (2014) demuestran que la mejor calidad fisiológica de la semilla en chiles se obtiene cuando se extrae la semilla de frutos maduros y almacenados por 15 días. No obstante, a partir de la etapa de inicio de cambio de color del fruto con extracción posterior es posible obtener semillas vigorosas y con germinación uniforme, que podrían usarse es programas de mejoramiento genético con ciclos de selección más cortos, que también servirían para la producción de semilla comercial pues son de suficiente calidad germinativa y se cosecharían en menos tiempo. Las semillas extraídas inmediatamente germinaron en promedio de 4 días después de un reposo de 15 días dentro del fruto y con valores menores de germinación total acumulada.

En los chiles de Árbol, Ancho y Guajillo, el extracto posterior de semilla produjo una germinación más rápida, con valores de 93, 99 y 98\%, respectivamente. En los tres tipos de chile la máxima velocidad de germinación ocurrió entre los 6 y los 11 d, independientemente de la combinación de tratamientos. Estos datos coinciden con lo realizado en el presente proyecto, donde se cosecharon los frutos al presentar 50\% de madurez fisiológica y posteriormente, se tuvieron en almacenamiento por un lapso de 12 a 15 días hasta que llegaron a su punto óptimo de madurez y así, se procedió a extraer la semilla y almacenarla por un periodo de cuatro meses.

Por su parte, Andueza et al. (2017) mencionan que la germinación de semilla de las variedades H$228, \mathrm{H}-259$ y H-244 fue significativamente igual con valores de $93 \%, 91 \%$ y $81 \%$, respectivamente, al ser sometidas a tres meses de almacenamiento; mientras que a seis meses la germinación de la variedad de H-259 subió a 93\%. Seis meses de almacenamiento de chile H-259 son suficientes para alcanzar la germinación máxima. El bioensayo desarrollado por Garruña et al. (2014) demostró que almacenar por 4 y 8 meses a $22{ }^{\circ} \mathrm{C}$ la semilla de chile Habanero disminuye la germinación a $70 \%$ y $0 \%$, cuando inicialmente fue de $95 \%$.

Los resultados también enfatizan la relación estrecha entre germinación y vigor de las variedades locales sobresalientes, pues formaron mayor número de plántulas normales de chile habanero. El ABA acelera la germinación y la emergencia. Las soluciones osmóticas de $\mathrm{KNO}_{3}$ y polietilen glicol 
(PEG) impiden la germinación durante el acondicionamiento y causan aumento de la emergencia. Las soluciones acondicionadoras de $\mathrm{KNO}_{3}$, PEG y ABA son las más adecuadas para obtener plántulas de chile habanero listas para el trasplante.

En la evaluación de las variables evaluadas en el a través del análisis de componentes principales (CP), para el caso de la fertilización, los dos primeros componentes principales refirieron a 87.07\% de la dispersión de datos. El CP1, explica $66.98 \%$ de la variabilidad total del experimento, representado en el eje de las abscisas y conformado por los porcentajes de germinación a los 8 y 16 días. El CP2 (eje de las ordenadas) observó $20 \%$ y donde destacan el peso de fruto y grosor del pericarpio. Así, en el primer cuadrante se ubican la fertilización química (100-0) y 25\% orgánico con $75 \%$ químico, los cuales sobresalieron por mejorar su germinación (a los 8 y 16 días) pero presentaron también bajos pesos y número de lóculos por fruto.

En seguida en los cuadrantes II y III, se ubicaron los tratamientos de fertilización totalmente orgánica (0-100) y $75 \%$ orgánica con $25 \%$ química, los cuales presentaron los más bajos porcentajes de germinación (a los 8 y 16 días), con la diferencia de que este último presentó un mayor peso de fruto y número de lóculos. Finalmente, en el cuadrante IV se ubicó el tratamiento 50-50 el cual permitió la mayor germinación a los 8 y 16 días, además del mayor peso de fruto y número de lóculos (Figura 1).

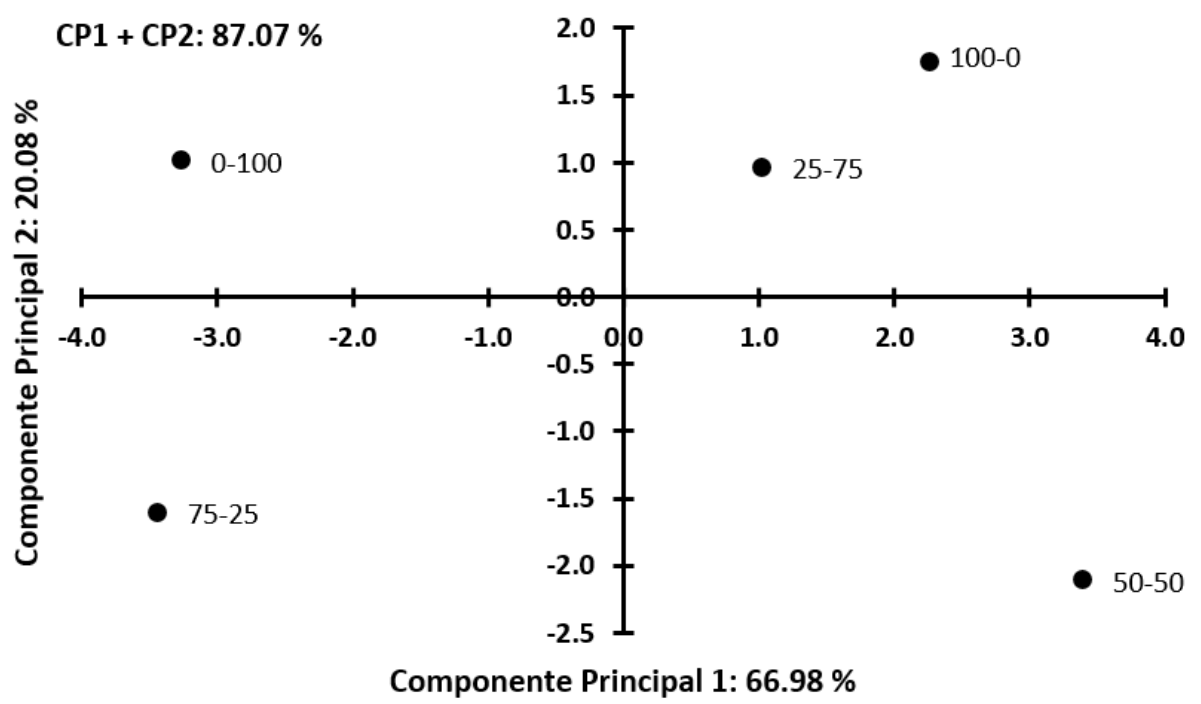

Figura 1. Componentes principales para tratamientos de fertilización química y orgánica en la producción de semillas de chile habanero. Roque, 2018.

En el caso de genotipos (Figura 2), no se observa algún agrupamiento por lo que la respuesta fue contrastante entre ellos. En este caso, los dos componentes principales de mayor importancia explican $100 \%$ de la variabilidad generada en el experimento. En el componente principal 1 en el eje de las X, que explica $94.2 \%$ de toda la dispersión, las variables número de lóculos por fruto, porcentaje de germinación a los 8 días y peso de fruto. A través de este componente, se encuentran los genotipos Rojo Campeche-12 (G4) y Naranja Yucatán-14 (G19) y finalmente G20 en cuyo mismo orden presentaron de mayor a menor número de lóculos, vigor (germinación a los 8 días) y peso de fruto. 


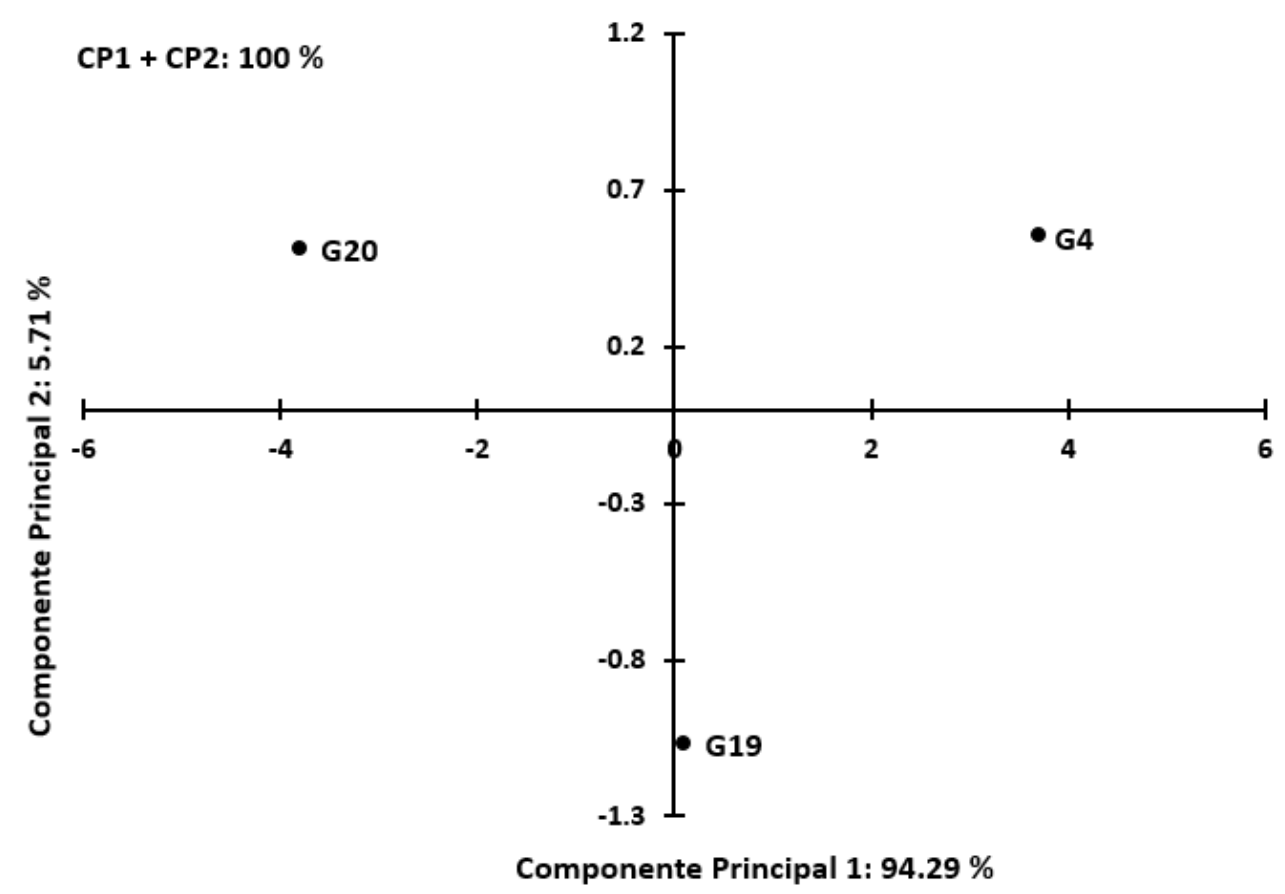

Figura 2. Componentes principales para genotipos de chile habanero. Roque, 2018.

Mientras que el componente principal 2, representado en el eje de las Y, que explica 5.7\% de las diferencias, se encuentra marcando pauta el grosor del pericarpio, siendo G4 y G20 los que presentaron el mayor valor, donde G19 presentó el menor valor. Al ser contrastantes los genotipos entre sí, el estadístico de los componentes principales señala que G4 es el único genotipo que es diferente y sobresale al resto de los materiales genéticos. México es considerado centro de origen del género Capcicum annuum y actualmente reconocido el chile habanero (Capcicum chinense) como denominación de origen de la península de Yucatán, esto indica, la gran variación genética de estas especies en el país.

Dentro de los componentes principales donde se agrupan todas las características evaluadas se encontró dispersión entre ellos para los genotipos y fertilizaciones evaluadas; es decir, las características cualitativas y cuantitativas difieren entre poblaciones de chile habanero. Al respecto, López et al. (2018) en un estudio de diversidad genética en chile habanero indican que la diversidad genética en poblaciones de chile habanero es alta, en donde $95.5 \%$ de la variación observada se encuentra dentro de las poblaciones y sólo $4.5 \%$ entre ellas. Se detectó un alto flujo génico entre las poblaciones.

Por su parte, Pacheco et al. (2016) midieron 13 características fenotípicas en 27 colectas pertenecientes a siete morfotipos de chiles nativos en Sinaloa y Nayarit. Los caracteres medidos fueron: altura de la planta, longitud de la rama, diámetro del tallo, longitud de la hoja, ancho de la hoja, longitud del pedúnculo, longitud del fruto, ancho del fruto, peso del fruto, espesor de la pared del fruto, peso de semillas por fruto, número de semillas por fruto y peso de la semilla. Los datos morfológicos fueron examinados mediante análisis univariados, análisis de varianza anidados y análisis de componentes principales. Se encontró amplia variabilidad en todas las características medidas. 
Los análisis de varianza anidados y de componentes principales diferenciaron los morfotipos, indicando una elevada variación entre ellos. Estos resultados indican que cada morfotipo es una entidad fenotípica y genética. Los datos mostrados son similares a los obtenidos en el presente trabajo, coincidiendo variantes como longitud del tallo (altura de la planta), peso del fruto y número de semillas por fruto, que en el componente principal 1, explican $94.2 \%$ de las variaciones totales, para el caso de genotipos. Latournerie et al. (2015) evaluaron doce poblaciones criollas de chile habanero en tres localidades de Yucatán, México y concluyen que las poblaciones fueron influenciadas por el ambiente (localidad), pero aun así encontraron materiales estables para rendimiento y otros muy específicos.

De igual forma, Ramírez et al. (2016) de las 11 poblaciones evaluadas de chile piquín, encontraron que el componente principal 1 explicó $25.6 \%$ de la variabilidad total, estuvo mejor correlacionado con las características de fruto (peso, ancho, longitud, días a fructificación y periodo de fructificación). CP2 que explicó $16.7 \%$ de la variabilidad total, estuvo mejor correlacionado con características de planta (ancho de hoja, pubescencia de hoja, pubescencia de tallo, hábito de crecimiento y macollamiento) y juntos explican $42.3 \%$ de la variabilidad total.

Por su parte, Guillén et al. (2016) encontraron diferencias estadísticas significativas $(p \leq 0.01)$ en nueve variables de chile perón. Los caracteres con la más alta variación entre colectas fueron el color de fruto, manchas o rayas presentes, forma fruto, ápice, base, longitud y diámetro de fruto, espesor de la pared y el número de semillas. El análisis de $\mathrm{CP}$ practicado indicó que los tres primeros componentes explicaron $62.95 \%$ de la variación total. Adicionalmente, el análisis de conglomerados realizado para las 13 variables, generó tres grupos de accesiones a una distancia euclidiana de 5.44. Algunas de estas variables, como lo es el caso del grosor del pericarpio (espesor de la pared) y el número de semillas, coinciden con el análisis componentes principales del presente proyecto, donde el CP1 explicó $66.98 \%$ de la variabilidad total de los tratamientos.

\section{Conclusiones}

Se encontró variabilidad entre las tres poblaciones de chile de chile habanero y una respuesta diferencial a la fertilización química y orgánica para las variables en estudio. Con el tratamiento 50-50, se obtuvo la mejor calidad de fruto (peso, número de lóculos y grosor de pericarpio), el mayor número de semillas y calidad fisiológica de éstas (germinación); tratamiento que igualó al de fertilidad $100 \%$ inorgánico en el número de semillas, grosor de pericarpio, germinación a los 8 y 16 días, pero lo superó estadísticamente en el peso de fruto $(3.6 \%)$, número de lóculos fruto ${ }^{-1}$ (10.3\%) y germinación (3\%).

La mejor colecta fue el genotipo Rojo Campeche 12 al sobresalir en todas las variables evaluadas, con un comportamiento promedio del peso de fruto de $4.8 \mathrm{~g}, 27.5$ semillas por fruto, 2.97 lóculos por fruto, un grosor del pericarpio del fruto de $0.17 \mathrm{~mm}, 27.5$ semillas por fruto y una germinación a los 16 días de 39.20\%, con la fertilización 50-50.

Lo anterior, permite explorar dosis y oportunidades de aplicación de abonos orgánicos que mejoren la producción de semilla con manejo sustentable y amigable para el ambiente, así como una mayor cantidad de colectas que expresen su potencial genético en diferentes ambientes en cuanto a la cantidad y oportunidad en la aplicación de fertilizantes orgánicos, independientemente de un mayor rango de las condiciones atmosféricas. 


\section{Literatura citada}

Andueza, N. R. H.; Latournerie, M. L.; Moran, V. N.; Cervantes, O. F.; Mendoza, E. M. y Rangel, L. J. A. 2017. Respuesta fisiológica de la semilla de chile habanero (Capsicum chinense Jacq.) al almacenamiento. Rev. Acta Universitaria. 27(6):3-10.

Ayala, V. M. J.; Ayala, G. O. J.; Aguilar, R. V. H. y Corona, T. T. 2014. Evaluación de la calidad de semilla de Capsicum annum L. durante su desarrollo en fruto. Rev. Fitotec. Mex. 37(1):79-87.

Borges, G. L.; Moo, K. C.; Ruíz, N. J.; Osalde, B. M.; González, V. G.; Yam, Ch. C. and Can, P. F. 2014. Soils used for habanero chili production in Yucatán: predominant physical and chemical characteristics. Agrociencia. 48(1):347-359.

DOF. 2010. Diario Oficial de la Federación. Declaratoria General de Protección de la Denominación de Origen Chile Habanero de la Península de Yucatán. http://dof.gob. $\mathrm{mx} /$ nota_detalle.php?codigo $=5145315 \&$ fecha $=04 / 06 / 2010$.

DOF. 2012. Diario Oficial de la Federación. Norma Oficial Mexicana NOM-189-SCFI-2012. Chile Habanero de la Península de Yucatán (Capsicum chinense Jacq.)-especificaciones y métodos de prueba. http://www.dof.gob. mx/normasOficiales/4730/seeco2/seeco $2 . h t m$.

Ertani, A. 2015. The use of organic biostimulants in hot pepper plants to help low input sustainable agriculture Chemical and biological technologies in Agriculture. 2-11 pp.

Garruña, H. R.; Latournerie, M. L.; Ayala, G. O.; Santamaría, J. M. y Pinzón L. L. 2014. Acondicionamiento pre-siembra: una opción para incrementar la germinación de semillas de chile Habanero (Capsicum chinense Jacq.). Agrociencia. 48(4):413-423.

Guillén, A. H.; Escalera, O. A. K.; Raya, M. Y. A.; Coria, M. R.G.; Lara, C. M. B.; Martínez, E.; López, G. Y.; Tajimaroa, Q. E.; Ramírez, G. L. y Valdivia, B. R. 2016. Estudio de la variabilidad del chile perón (Capsicum pubescens) en Michoacán, México. Acta fitogenética. 3(1):1-204.

Herrera, A. A.; Cervantes, O. F.; Antuna, G. O.; García, R. J. G.; Rodríguez, M. D.; Rodríguez, H. S.A.; Andrio, E. E. y Mendoza, E. M. 2018. Deterioro de la calidad de la semilla de chile piquín de cuatro colectas de Querétaro y Guanajuato. Rev. Mex. Cienc. Agric. 9(8):1627-1638.

Huez, L. M. 2013. Productividad de chile habanero (Capsicum chinense Jacq.) bajo condiciones de invernadero en la costa de Hermosillo. In: XVI Congreso Internacional de Ciencias Agrícolas. 282-286 pp.

ISTA. 2004. International Seed Testing Association. International rules for seed testing. Rules 2004. ISTA Editions. Bassersdorf, CH-Switserland. 243 p.

Latournerie, M. L.; López, V. J.; Castañón, N. G.; Mijangos, C. J. O.; Espadas, V. G.; Pérez, G. A. and Ruiz, S. E. 2015. Agronomic evaluation of habanero pepper (Capsicum chinense Jacq.) germplasm. AP Agroproductividad. 12(2):24-29.

López, A. M.; Poot, M. J. E. and Mijangos, C. M. A. 2012. Response of habanero pepper (Capsicum chinense L. Jacq) organic fertilizer supply in Tabasco, México. Revista Científica UDO Agrícola. 12(2):307-312.

López, E. S. T.; Latournerie, M. L.; Castañón, N. G.; Ruiz, S. E.; Gómez, L. J. F.; Andueza, N. R. H y Mijangos, C. J. O. 2018. Diversidad genética de chile habanero (Capsicum chinense Jacq.) mediante SSR. Rev. Fitotec. Mex. 41(3):227-236. 
López, G. J. D.; Villegas, T. O. G.; Sotelo, N. H.; Andrade, R. M.; Juárez, L. P. y Martínez F. E. 2017. Rendimiento y calidad del chile habanero (Capsicum chinense Jacq.) por efecto del régimen nutrimental. Rev. Mex. Cienc. Agríc. 8(8):1747-1758.

Nieves, G. F.; Alejo, S. G. and Luna, E. G. 2013. Techniques for the sustainable management of the production of the habanero chili (Capsicum chinense Jacq.) Rev. Bio Ciencias. 2(3):98-101.

Pacheco, O.; Hernández, V. A.; Parra, T. S.; Valdés, O. S. y Osuna E. T. 2016. Exploración y diversidad morfológica in situ de chiles nativos de Sinaloa y Nayarit. Acta Fitogenética, 3(1):1-219.

Quintal, O. W. C.; Pérez, G. A.; Latournerie, M. L.; May, L. C.; Ruíz, S. E. y Martínez, C. A. J. 2012. Uso del agua, potencial hídrico y rendimiento de chile habanero. Rev. Fitotec. Mex. 35(2):155-160.

Ramírez, C. R.; López, M. J. D.; Troyo, D. E.; Gallegos, R. M. A.; Vázquez, V. C.; Ramírez, I. J. A. y García, H. J. L. 2016. Determinación preliminar y de normas e interacciones nutrimentales en chile ancho (Capsicum annum L.) en la Comarca Lagunera. Nova Scientia, 8(2):198-218.

Ramírez, M. M.; Arcos, C. G.; Mata, V. H. y Vázquez, G. E. 2012. Jaguar, variedad de chile habanero para México. Centro de Investigación Regional del Noreste. Campo Experimental Las Huastecas. Folleto técnico. 35 p.

Reyes, R. A.; López, A. M.; Ruiz, S. E.; Latournerie, M. L.; Pérez, G. A.; Lozano, C. M. G. and Zavala, L. M. J. 2014. Effectiveness of microbial inoculants on growth and productivity of habanero pepper (Capsicum chinense Jacq.) Agrociencia. 48(3):285-294.

SAS. 2003. Statistical Analysis System. SAS Version 9.3. Institute, Inc. Cary, NC, USA.

SIAP. 2015. Servicio de Información Agroalimentaria y Pesquera. SAGARPA. Márgenes de comercialización de chile habanero. Boletín. https://www.gob.mx/siap.

SNICS. 2019. Servicio Nacional de Inspección y Certificación de Semillas. https://www.gob.mx/snics/documentos/catalogo-nacional-de-variedades-vegetales.

SNICS. 2019. Servicio Nacional de Inspección y certificación de semillas. Guía técnica del chile habanero. https://www.researchgate.net/publication/284787914_Chile_habanero_ Capsicum_chinense_Jacq_Guia_tecnica_para_la_descripcion_varietal.

Soria, F. M.; Trejo, J. A.; Tun, S. J. M. y Terán, S. R. 2002. Paquete tecnológico para la producción de chile habanero (Capsicum chinense Jacq.). Instituto Tecnológico Agropecuario No. 2. Conkal, Yucatán. 128 p.

Tamayo, M. J.; Martínez, O. E.; Monforte, M. G.; Munguía, G. I. and Ruiz, M. A. 2014. Agroecology as proposed model applied to the habanero pepper growing crop in Peto, Yucatan. Rev. Mex. Agroneg. 35(1):969-978.

Tucuch, H. C. J.; Alcántar, G. G.; Ordaz, C. V. M.; Santizo, R. J. A. y Larqué, S. A. 2012. Producción y calidad de chile habanero (Capsicum chinense Jacq.) con diferentes relaciones de $\mathrm{NH}_{4}{ }^{+} / \mathrm{NO}_{3}{ }^{-}$y tamaño de partícula de sustratos. Terra Latinoam. 30(1):9-15. 